\title{
Video capsule endoscopy as a tool for evaluation of obscure overt gastrointestinal bleeding in the intensive care unit
}

\section{다(1) $(\odot)$}

\author{
Authors \\ Institutions \\ 1 Department of Medicine, UMass Memorial Medical \\ Center, Worcester, MA \\ 2 Division of Gastroenterology, UMass Memorial Medical \\ Center, Worcester, MA \\ 3 Division of Data Sciences and Technology, Information \\ Technology, UMass Medical School, Worcester, MA
}

Shahrad Hakimian ${ }^{1}$, Salmaan Jawaid ${ }^{2}$, Yurima Guilarte-Walker ${ }^{3}$, Jomol Mathew ${ }^{3}$, David Cave ${ }^{2}$

submitted 4.12 .2017

accepted after revision 21.2.2018

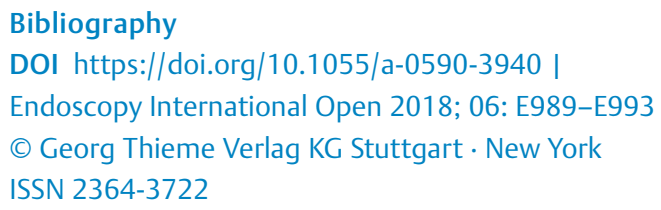

Corresponding author

Shahrad Hakimian, MD, UMass Memorial Medical Center, Department of Internal Medicine, 55 Lake Avenue North, Worcester, MA 01655, United States

Fax: +1-508-8563981

shahrad.hakimian@umassmemorial.org

\section{ABSTRACT}

Background and study aims Video capsule endoscopy (VCE) is a minimally invasive tool that helps visualize the gastrointestinal tract from the esophagus to the right colon without the need for sedation or preparation. VCE is safe with very few contraindications. However, its role and safety profile in the intensive care unit (ICU) population have not been reported. The aim of this study is to evaluate the safety, efficacy, and feasibility of VCE use in ICU patients. Patients and methods We conducted a single-center retrospective observational study of patients who underwent VCE for evaluation of obscure overt gastrointestinal bleeding in the ICU between 2008 and 2016.

Results This study included 48 patients who were admitted to the UMass Memorial Medical Center ICUs for gastrointestinal bleeding. VCE was successfully completed in 43/ $48(90 \%)$ patients. The entire length of small bowel could be evaluated in $75 \%$ and the source of bleeding was identified in $44 \%$ of the patients. The most commonly identified source of bleeding included small bowel angioectasias, duodenal erosions/ulcers, and small bowel polyps. No major complications could be attributed to the VCE. Only 1 capsule was retained after $2 \mathrm{wk}$; however, there was no incidence of bowel obstruction, perforation, or capsule aspiration.

Conclusions This observational retrospective study demonstrates that VCE may be a safe, feasible, and effective diagnostic tool in evaluation of gastrointestinal bleeding in the ICU population with few complications. VCE may be a safe diagnostic prelude and be a guide to the correct therapeutic procedure if needed, in the context of patients who are seriously ill.

\section{Introduction}

Patients with severe gastrointestinal bleeding (GIB) who are hemodynamically unstable are usually admitted to an intensive care unit (ICU) [1] for stabilization and possible endoscopic management. By definition, these patients are usually hemodynamically unstable and are at increased risk of endoscopic complications during evaluation [2-6]. Hematemesis localizes bleeding to within reach of a gastroscope. In patients with nonhematemesis GIB, the presence of melena or hematochezia has little localizing value [6], leading to what has become a traditional approach of upper endoscopy followed by colonoscopy and other procedures if 1 of those 2 are not diagnostic [7]. However, the yield of conventional endoscopic procedures in this context is known to be low. An early diagnostic/endoscopic approach is thought to have higher diagnostic yield in some studies, yet this advantage needs to be balanced with risk of endoscopic complications [8-11].

Video capsule endoscopy (VCE) is a minimally invasive tool that can visualize the gastrointestinal tract from esophagus to the right colon without the need for sedation or preparation. We hypothesize that VCE could serve as an alternative to the traditional approach to GIB in an acutely unstable or high-risk patient. VCE has been investigated as a triaging tool in the 
emergency room to determine the population of patients who would benefit from additional diagnostic and therapeutic interventions [12]. However, before the utility of VCE as first-line evaluation of GIB in ICU patients can be established, the safety and efficacy of this technique in ICU patients need to be evaluated further. We therefore investigated the safety and efficacy of VCE use in the ICU in a retrospective case series of patients admitted to the ICU with obscure overt GIB. To our knowledge, there are no prior studies that specifically evaluated the role and safety profile of VCE in the ICU population for evaluation of GIB.

\section{Patients and methods}

We conducted a single-center retrospective observational study (case-series) of patients who underwent VCE for evaluation of obscure overt GIB in our medical, cardiac, and surgical ICUs between 2008 and 2016 at UMass Memorial Medical Center, a large tertiary referral center in central Massachusetts. Inclusion criteria included age more than $18 \mathrm{y}$, a diagnosis of GIB during that admission, and VCE during an ICU stay. Patients were identified through billing codes, and data collected included patients' demographics, comorbidities, VCE results, additional diagnostic/therapeutic procedures, and laboratory data. Standard descriptive statistics were used for demographic data, VCE findings, and complications using Microsoft Excel 2007 (Microsoft, Redmond, WA, USA). Additionally, we used Student's t-test for comparing continuous variables, and chisquared test was used for binary variables. The patients were investigated using either the $\mathrm{M}_{2} \mathrm{~A}$ or PillCam SB2 capsules ( $\mathrm{G}$ venImaging, Jocqneam, Israel). The capsules were read on a workstation using Rapid software versions 3 to 8. All VCE images were read by expert endoscopists with many years of experience in interpreting VCEs. All patients had been screened for contraindications of VCE including but not limited to history of bowel obstruction. In a few patients with high risk of gastric retention (e.g., prior history) or evidence of retention on the real-time viewer, a dose of intravenous metoclopramide or erythromycin was administered before or during the capsule study. VCE was deemed successful if the entire length of the small bowel could be evaluated or if significant length of the small bowel was evaluated without significant device malfunction, missing frames, or gastric retention. The retrospective chart review was approved by the UMass Memorial Institutional Review Board.

\section{Results}

This study included 48 patients who were admitted to the ICU for GIB and who had negative esophagogastroduodenoscopy (EGD) and in many cases colonoscopy (69\%) and were categorized as having obscure overt GIB on further investigation. Average age of patients was $70 \pm 16.5$ y with $58 \%$ male ( $\triangleright$ Table 1 ). The majority of patients ( $92 \%$ ) were Caucasian. More than half (56\%) were on mono or dual antiplatelet therapy, and $31 \%$ were anticoagulated. About one-fifth of patients (19\%) had international normalized ratio (INR) $>3$, and $29 \%$ required $2 \mathrm{~L}$ or more
- Table 1 Demographics.

\begin{tabular}{|l|c|}
\hline & $\mathbf{n}(\%)$ \\
\hline Male & $28(58)$ \\
\hline Female & $20(42)$ \\
\hline Age $(y)$ & $70.4 \pm 16.5$ \\
\hline BMI & $28.3 \pm 7.5$ \\
\hline Caucasian & $44(92)$ \\
\hline Current smoker & $13(27)$ \\
\hline Former smoker & $26(54)$ \\
\hline Alcohol use & $15(31)$ \\
\hline NSAIDs use & $5(10)$ \\
\hline Aspirin alone & $22(46)$ \\
\hline Dual antiplatelet therapy & $5(10)$ \\
\hline Anticoagulated & $15(31)$ \\
\hline
\end{tabular}

Presenting symptom

\begin{tabular}{|l|c|}
\hline Hematemesis & $6(13)$ \\
\hline Hematochezia & $15(31)$ \\
\hline Melena & $23(48)$ \\
\hline Anemia & $4(8)$ \\
\hline Comorbidities & \\
\hline
\end{tabular}

\begin{tabular}{|l|c|}
\hline Coronary artery disease & $28(58)$ \\
\hline Recent MI & $4(8)$ \\
\hline COPD & $11(23)$ \\
\hline
\end{tabular}

Pneumonia $8(17)$

Severe aortic stenosis $6(13)$

\begin{tabular}{l|l}
$\mathrm{CHF}$ & $13(27)$
\end{tabular}

\begin{tabular}{l|l}
\hline ESRD & $6(13)$ \\
\hline
\end{tabular}

\begin{tabular}{l|l} 
Cirrhosis & $7(15)$
\end{tabular}

History of IBD $0(0)$

\begin{tabular}{|l|l|}
\hline Prior abdominal surgeries & $23(48)$ \\
\hline
\end{tabular}

$\begin{array}{ll}\text { History of small bowel obstruction } & 0(0)\end{array}$

\begin{tabular}{|l|c|}
\hline INR $>3$ & $9(19)$ \\
\hline Hypoxia (>2 L NC) & $14(29)$ \\
\hline Vasopressors required & $3(6)$ \\
\hline Need for transfusion & $45(94)$ \\
\hline Lowest HGB (average) & $6.39 \pm 1.14$ \\
\hline Lowest HGB (range) & $3.8-8.5$ \\
\hline Units of blood transfused & $5.9 \pm 4.8$ \\
\hline
\end{tabular}

BMI: body mass index; COPD: chronic obstructive pulmonary disease; CHF: congestive heart failure; ESRD: end-stage renal disease; IBD: inflammatory bowel disease; MI: myocardial infarction; NSAID: nonsteroidal anti-inflammatory drug; HGB: hemoglobin; NC: nasal canula 
of supplemental oxygen. Three patients required vasopressor support for hypotension. Twenty-three patients (48\%) initially presented with melena, $15(31 \%)$ with hematochezia, and 6 $(13 \%)$ with hematemesis. The most common underlying comorbidities included coronary artery disease, congestive heart failure, and chronic obstructive pulmonary disease. Nearly half of patients ( $48 \%$ ) had history of intra-abdominal surgery, but none had history of prior small bowel obstruction, inflammatory bowel disease, or fistulas. The mean lowest hemoglobin was $6.39 \pm 1.14 \mathrm{mg} / \mathrm{dL}$ (range: $3.8-8.5 \mathrm{mg} / \mathrm{dL}$ ) on or within $2 \mathrm{~d}$ of admission. Almost all patients 45 (94\%) required blood transfusions with a mean of $5.8 \pm 4.8$ units transfused. Transfusion requirements were higher in patients whose bleeding source could be identified as compared to those with unidentified source $(7.9 \pm 5.7$ vs. $4.3 \pm 4.8$ units transfused, $P<0.01)$. The mean lengths of hospital and ICU stays were $10.4 \pm 9.6 \mathrm{~d}$ and $5.5 \pm 3.6 \mathrm{~d}$, respectively.

Most patients $44 / 48$ (92\%) had EGD within $24 \mathrm{~h}$ of admission. Two patients had prior negative EGDs within the past 30 $\mathrm{d}$, and 1 patient had EGD at day 8 of admission due to presentation with hematochezia on day 1 and repeat bleeding on day 7 . Average time to colonoscopy was $1.9 \pm 0.9 \mathrm{~d}$ in the two-thirds of patients who underwent this procedure ( $\triangleright$ Table 2$)$. One patient with prior history of small bowel bleeding underwent VCE as first-line evaluation of GIB. Sixteen patients (33\%) underwent VCE as second-line evaluation. The remainder of patients underwent VCE as third- or fourth-line evaluation for obscure overt GIB. VCE was successfully completed in 43 /48 (90\%) patients. The majority of capsules were swallowed by the patient (75\%) while the other $25 \%$ were placed endoscopically via EGD, most commonly during negative EGD as the next step in the patient's evaluation ( $>$ Table 2 ). The entire length of small bowel could be evaluated in 36/48 (75\%). The source of bleeding was identified in $21 / 48$ (44\%) patients. However, the source was identified more commonly when VCE was performed within $48 \mathrm{~h}$ of suspected bleeding ( $63 \%$ within $48 \mathrm{~h}$ vs. $31 \%$ with VCE after $48 \mathrm{~h}, P=0.03)$. The most commonly identified source of bleeding included small bowel angioectasias, duodenal erosions/ulcers, and small bowel polyps. The most commonly identified incidental lesions in the small bowel included angioectasias (nonbleeding and no stigmata of recent bleeding, portal hypertensive gastropathy/small bowel varices, and erosions. Additional diagnostic interventions (tagged Red Blood Cell scan, angiography, and deep enteroscopy) was performed in $17 \%$ of patients while $23 \%$ required additional therapeutic interventions.

No major complications could be attributed to the VCE. Only 1 capsule was retained on abdominal imaging after $2 \mathrm{wk}$; however, there was no incidence of bowel obstruction, perforation, or capsule aspiration ( $>$ Table 3 ). Two additional capsules did not pass through the stomach to enter the small bowel within the $8 \mathrm{~h}$ of recorded images but were excreted on follow-up imaging within 2 wk. Two earlier studies also included missing frames or poor image quality within the small bowel, which limited the interpretation of the small bowel images.
- Table 2 Outcomes.

\begin{tabular}{|l|l|}
\hline & $\mathbf{n}(\%)$ \\
\hline Successful completion of VCE & $43(90)$ \\
\hline Entire small bowel visualized & $36(75)$ \\
\hline Anatomic source of bleeding identified & $21(44)$ \\
\hline - VCE within 48 & $12 / 19(63)$ \\
\hline - VCE after 48h & $9 / 29(31)$ \\
\hline - p-value & 0.03 \\
\hline Actively bleeding lesions & $9(19)$ \\
\hline
\end{tabular}

Common sources of bleeding identified

$\begin{array}{ll}\text { Angioectasias } & 10(21)\end{array}$

\begin{tabular}{|l|l|}
\hline Small intestinal erosions/ulcers & $2(4)$ \\
\hline Small bowel polyps & $2(4)$ \\
\hline Portal hypertensive gastropathy & $2(4)$ \\
\hline Other & $5(11)$ \\
\hline
\end{tabular}

Most common incidental findings

Angioectasias (not clear source of bleed)

$7(15)$

Portal hypertensive gastropathy / small intestinal varices

Erosions

$2(4)$

Mode of capsule delivery

Swallowed capsule

Endoscopically placed

$12(25)$

Timing of initial diagnostic procedures

EGD within $24 \mathrm{~h}$

$44(92)$

Prior EGD within $30 \mathrm{~d}$

$2(4)$

Colonoscopies during the same admission

$33(69)$

Days to colonoscopy (d)

$1.9 \pm 0.9$

Capsule as first-line

1 (2)

Days to VCE (d)

$3.47 \pm 2.3$

Need for additional procedures

$19(40)$

Diagnostic

8 (17)

Therapeutic

$11(23)$

\section{Discussion}

This retrospective case series demonstrates that VCE may be a safe, feasible, and effective diagnostic tool in the evaluation of obscure overt GIB in the critically ill ICU population. The overall success rate of the procedure was $90 \%$, with the source of bleeding identified in nearly half of the patients. Almost all patients in this study had obscure overt GIB and were previously evaluated via other modalities. There were no major complications attributed to the VCE. 
- Table 3 Safety data.

Major complications

$0(0)$

Minor complications

$1(1)$

Capsule retention at 2 wk

$1(1)$

Aspiration

$0(0)$

Bowel obstruction

$0(0)$

Length of hospital stay (d)

$10.4 \pm 9.5$

Length of ICU stay (d)

$5.5 \pm 3.6$

Need for intubation for GIB or tracheostomy

Recurrence of bleeding at 6 mo

$6(12.5)$

$12(25)$

While the current study does not address the utility of VCE as a first-line diagnostic tool for evaluation of GIB, as a minimally invasive tool, VCE may be a safe diagnostic alternative to standard endoscopic procedures in patients at risk for significant complications from the more invasive procedures, such as those with elevated INR, hypoxemia, or recent myocardial infarction [3]. This study demonstrates that VCE is safe in the most critically ill patients with obscure overt GIB who do not otherwise have other major contraindications to VCE. However, the role of VCE as the first-line diagnostic modality in this setting needs to be evaluated further. Inpatient data shows the earlier VCE is used the better the diagnostic yield [8]. This observation is supported by similar findings in the current study as patients who underwent VCE within $48 \mathrm{~h}$ of admission had twice the likelihood of bleeding source identification. Larger prospective studies, particularly with early deployment, are necessary to further evaluate the safety and efficacy of this tool in these settings.

Newer video capsule models allow live visualization of the upper gastrointestinal tract in real time to determine the presence of active bleeding in the esophagus or stomach and confirm passage of capsule into the small intestine. This may help stratify high-risk patients regarding urgency of more definite therapeutic interventions while offering additional diagnostic information regarding possible sources of bleeding in the small intestine or presence of blood in the right colon. This can, in turn, potentially minimize the number of invasive procedures, decrease the length of hospitalization, and minimize complications in the critically ill patients.

In our cohort, we observed a few cases of gastric retention of the capsule and delayed transit. Other published and unpublished studies confirm the occurrence of delayed transit in bed-ridden patients, which are common in the ICU setting. Several medications including opioids, commonly used in the ICU, may also alter gut motility and lead to delayed gastric emptying. The only other study of VCE in ICU patients, to our knowledge, investigated transit time of the VCE in a small cohort of ICU patients [13]. While this study was not able to show a statistically significant difference in transit time between ICU patients compared to ambulatory patients (most likely due to the very small sample size), there was a large degree of variability in transit times in the critically ill population. In our practice, we have most recently developed an algorithm for passage into the small intestine for swallowed capsules at about an hour after capsule ingestion. If the capsule is not in the small intestine at that point, we have used prokinetic agents such as metoclopramide or erythromycin with good success. This increases the yield of VCE in such select patients.

Another rare technical difficulty was missing frames or poor image quality in 2 (4\%) of our earlier patients. This is most likely due to electrical interference from nearby devices, distance from the receiver, or the patient's excessive movement. Newer devices with higher-quality signals and images have mostly resolved these issues in our most recent cohorts of ICU and nonICU patients. However, wireless interference from ventricular assist devices on image quality of VCE continues to remain a limitation reported in the literature [14].

The main limitations of this study include the small sample size and the limitations of the retrospective design. The sample size is limited by the number of VCEs already performed at our institution, but to our knowledge, this is the largest cohort of VCEs studied in the ICU setting. A prospective design will increase the power of the study and make the study more generalizable. The retrospective design also adds its limitations as there is no control arm and neither the patient nor the clinicians are blinded. In addition, this study by its nature is self-selected for patients with obscure GIB. Therefore, further larger prospective studies are necessary before generalizing the result to the general ICU population with GIB. Other limitations may include variation in patient acuity and severity of disease. Our center is a large tertiary/quaternary with high-acuity patients, but further studies may help generalize the result to smaller community sites.

In summary, VCE is safe for use in ICU patients and may be useful in patients with GIB in detecting the site of active bleeding and providing guidance for the most appropriate therapeutic procedure, if needed.

\section{Competing interests}

Dr. Cave is an Olympus Corporation research grant recipient. Funding source: None

References

[1] Das AM, Sood N, Hodgin K et al. Development of a triage protocol for patients presenting with gastrointestinal hemorrhage: a prospective cohort study. Crit Care 2008; 12: R57

[2] Freeman ML, Hennessy JT, Cass OW et al. Carbon dioxide retention and oxygen desaturation during gastrointestinal endoscopy. Gastroenterology 1993; 105: $331-339$

[3] Cappell MS, lacovone FM Jr. Safety and efficacy of esophagogastroduodenoscopy after myocardial infarction. Am J Med 1999; 106: 29 35

[4] Amornyotin S. Sedation-related complications in gastrointestinal endoscopy. World J Gastrointest Endosc 2013; 5: 527-533 
[5] Enestvedt BK, Eisen GM, Holub J et al. Is the American Society of Anesthesiologists classification useful in risk stratification for endoscopic procedures? Gastrointest Endosc 2013; 77: 464-471

[6] Fine KD, Nelson AC, Ellington RT et al. Comparison of the color of fecal blood with the anatomical location of gastrointestinal bleeding lesions: potential misdiagnosis using only flexible sigmoidoscopy for bright red blood per rectum. Am J Gastroenterol 1999; 94: 3202 3210

[7] Early DS, Ben-Menachem T et al. Appropriate use of Gl endoscopy. ASGE Standards of Practice Committee Gastrointest Endosc 2012; 75: $1127-1131$

[8] Singh A, Marshall C, Chaudhuri B et al. Timing of video capsule endoscopy relative to overt obscure GI bleeding: implications from a retrospective study. Gastrointest Endosc 2013; 77: 761 - 766

[9] Corbett GD, Cameron EA. Timing of endoscopy in high-risk patients with nonvariceal upper gastrointestinal bleeding. Endoscopy 2011; 43: 925
[10] Yamada A, Watabe H, Kobayashi Y et al. Timing of capsule endoscopy influences the diagnosis and outcome in obscure-overt gastrointestinal bleeding. Hepatogastroenterology 2012; 59: 676-679

[11] Sonnenberg A. Timing of endoscopy in gastrointestinal bleeding. United European Gastroenterol J 2014; 2: 5 -9

[12] Sung JJ, Tang RS, Ching JY et al. Use of capsule endoscopy in the emergency department as a triage of patients with $\mathrm{Gl}$ bleeding. Gastrointest Endosc 2016; 84: 907 - 913

[13] Rauch S, Krueger K, Turan A et al. Determining small intestinal transit time and pathomorphology in critically ill patients using video capsule technology. Intensive Care Med 2009; 35: 1054 - 1059

[14] Amornsawadwattana S, Nassif M, Raymer D et al. Video capsule endoscopy in left ventricular assist device recipients with obscure gastrointestinal bleeding. World J Gastroenterol 2016; 22: 45594566 